\title{
Functional analysis and heterologous expression of bifunctional glutathione synthetase from Lactobacillus
}

\author{
Zhi-Qiang Xiong, ${ }^{*}$ Ling-Hui Kong, ${ }^{*}$ Guang-Qiang Wang, ${ }^{*}$ Yong-Jun Xia, ${ }^{*}$ Hui Zhang, ${ }^{*}$ Bo-Xing Yin,† \\ and Lian-Zhong $\mathrm{Ai}^{* 1}$ \\ *Shanghai Engineering Research Center of Food Microbiology, School of Medical Instrument and Food Engineering, \\ University of Shanghai for Science and Technology, Shanghai 200093, China \\ †Kangyuan Dairy Co. Ltd., Yangzhou University, Yangzhou 225004, China
}

\section{ABSTRACT}

Bifunctional glutathione synthetase (GshF) has recently been reported to simultaneously catalyze the 2-step ATP-dependent biosynthesis of reduced glutathione (GSH). In this work, 19 putative $g s h F$ were mined from the complete sequenced genome of 20 representative Lactobacillus species. To functionally analyze these putative GshF, GshF from Lactobacillus plantarum and Lactobacillus casei were selected and successfully expressed in Escherichia coli. Compared with the control without expressing GshF, GSH titers were enhanced significantly in E. coli with overexpression of GshF, demonstrating that putative GshF from Lactobacillus have functional activities on GSH biosynthesis. Moreover, with the expression of GshF from $L$. plantarum in E. coli as a paradigm, GSH yield (286.5 $\mu M)$ was strongly improved by $177.9 \%$ with optimized induced conditions and precursor concentration compared with the control under unoptimized conditions. Transcriptional analysis showed that key genes of endogenous GSH metabolism and precursor biosynthesis were remarkably suppressed by GshF expression, indicating that the increase of GSH titer was attributed to heterologous expression of GshF. Overall, our results suggested that gshF is enriched in Lactobacillus and that heterologous expression of GshF is an efficient strategy for improving GSH biosynthesis.

Key words: bifunctional glutathione synthetase, glutathione biosynthesis, heterologous expression, Lactobacillus

\section{INTRODUCTION}

The genus Lactobacillus, as a member of versatile probiotic lactic acid bacteria $(\mathbf{L A B})$, is widely present

Received November 16, 2017.

Accepted March 31, 2018

${ }^{1}$ Corresponding author: ailianzhong@hotmail.com in dairy products and other fermented foods, naturally inhabits the human environment, and has potential health-associated properties (Settachaimongkon et al., 2016; Xiong et al., 2017). Many species of Lactobacillus (e.g., Lactobacillus plantarum and Lactobacillus casei) have been commercialized in the probiotic market and widely applied in the food industry, such as milk fermentation and cheese production. With the advent of omics technologies, Lactobacillus have emerged as a group of model organisms for studies and research on LAB. For example, the whole-genome sequencing of L. plantarum WCFS1 isolated from human saliva has provided insight into potential probiotic properties such as adhesion and immunomodulation capacity (Kleerebezem et al., 2003; Siezen et al., 2012).

Reduced glutathione (GSH; $\gamma$-glutamyl-Lcysteinylglycine), a bioactive tripeptide consisting of L-Glu, L-Cys, and Gly, is one of the most abundant nonprotein thiol compounds in living organisms (Wang et al., 2016). Due to its physiological functions, including resistance to oxidative stresses, regulation of cellular redox homeostasis, and enhancement of host immunity (Yang et al., 2016), GSH is widely used in food, pharmaceutical, and cosmetic industries (Li et al., 2004). Typically, GSH is the ATP-dependent synthesis via 2 consecutively biosynthetic steps using soluble enzymes in the cytosol (Figure 1), $\gamma$-glutamylcysteine synthetase (also known as glutamate-cysteine ligase, EC 6.3.2.2; $\boldsymbol{\gamma}$-GCS) and GSH synthetase (EC 6.3.2.3, GS; Li et al., 2004). First, L-Glu and L-Cys are catalyzed by $\gamma$-GCS to form the dipeptide $\gamma$-L-glutamylL-cysteine, which subsequently connects with Gly by GS to form GSH (Yang et al., 2016). The first reaction catalyzed by $\gamma$-GCS is the rate-limiting step of GSH biosynthesis because of the feedback inhibition of GSH at both the transcriptional and posttranslational levels (Xiong et al., 2015). Moreover, GSH peroxidase (EC 1.11.1.9; GPx) and GSH reductase (EC 1.8.1.7; GR) are the key enzymes involved in GSH metabolism. The former catalyzes the conversion of reduced GSH into 


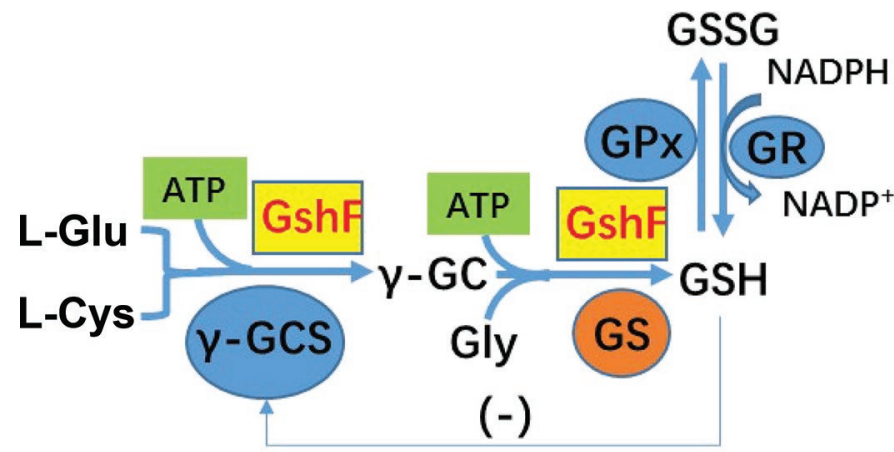

Figure 1. The simplified pathway of reduced glutathione (GSH) synthesis. $\gamma$-GCS $=\gamma$-glutamylcysteine synthetase; GS $=$ GSH synthetase; $\mathrm{GshF}=$ bifunctional GSH synthetase; $\mathrm{GPx}=\mathrm{GSH}$ peroxidase $\mathrm{GR}=\mathrm{GSH}$ reductase; GSSG $=$ GSH disulfide. Minus sign $(-)$ denotes feedback inhibition. Color version available online.

its oxidized form glutathione disulfide, and the latter enzyme regenerates GSH from glutathione disulfide for maintaining thiol-redox homeostasis in cells (Pophaly et al., 2017).

Reduced glutathione is widely distributed in eukaryotic microorganisms and gram-negative bacteria (Pophaly et al., 2012) but rarely in gram-positive bacteria except some low-GC-content bacteria (i.e., Streptococcus, Lactococcus, Lactobacillus, Enterococcus, Clostridium, and Listeria). Interestingly, some grampositive bacteria (e.g., Streptococcus thermophilus, Streptococcus agalactiae, and Enterococcus faecalis) have been reported to synthesize GSH, but no direct evidence shows the presence of either the activities of $\gamma$-GCS and GS or the genes GGCS and GS in the genome sequences of those strains (Li et al., 2005). Until recently, bifunctional GSH synthetase (GshF) was reported to simultaneously catalyze the 2-step ATPdependent biosynthesis of GSH in those strains because GSH biosynthesis generally requires the consecutive reaction by $\gamma$-GCS and GS (Vergauwen et al., 2006; Ge et al., 2012). Bifunctional GSH synthetase contains an N-terminal $\gamma$-GCS-like domain followed by a typical ATP-grasp GS-like domain, but this GS-like domain has no sequence homology with any known GS (Stout et al., 2012). Based on sequence alignment, more than 20 microbial species, most of which are gram-positive bacteria such as L. plantarum and S. thermophilus, have been found to contain GshF, of which most have not been studied by cloning and expression ( $\mathrm{Li}$ et al., 2011). Bifunctional GSH synthetase is normally insensitive to high concentrations of GSH. Hence, using GshF for GSH biosynthesis, GSH titer could be even higher by alleviating the feedback inhibition of GSH ( $\mathrm{Li}$ et al., 2011). In this work, the distribution of $g s h F$ in Lactobacillus was examined based on the complete sequenced genome of Lactobacillus. Biosynthesis of GSH was significantly improved in E. coli with heterologous overexpression of GshF from Lactobacillus. To our knowledge, it is still unclear how heterologous GshF affects exogenous metabolism in the host. Our study investigates the effect of heterologous GshF on exogenous GSH metabolism and GSH precursor biosynthesis in $E$. coli at the transcriptional level by real-time quantitative reverse transcription PCR (RT-qPCR) analysis.

\section{MATERIALS AND METHODS}

\section{Plasmids, Strains, Regents, and Culture Conditions}

All plasmids, strains, and primers in this study are listed in Tables 1 and 2. Escherichia coli Top10 was used as the host for plasmid construction. Escherichia coli BL21(DE3) was used for gene expression. Restriction enzymes and T4 DNA ligase were purchased from Takara Biomedical Technology Co. Ltd. (Beijing, China). The PrimeSTAR HS DNA polymerase (Takara) was used for DNA amplification. Kits for DNA fragment purification and plasmid extraction were provided by Axygen Biosciences (Corning Inc., Jiangsu, China). All primers and other reagents were purchased by Shanghai Sangon Biotech Co. Ltd. (Shanghai, China). LuriaBertani medium was used for plasmid construction and gene expression. According to the resistance marker of plasmids, kanamycin $(50 \mathrm{mg} / \mathrm{L})$ was added in each culture. Lactobacillus plantarum WCFS1 and L. casei

Table 1. Strains and plasmids used in this study

\begin{tabular}{|c|c|c|}
\hline Strain/plasmid & Relevant characteristics & Source/reference \\
\hline E. coli BL21(DE3) & $\begin{array}{l}\left.\mathrm{F}^{-} \text {ompT gal dcm lon hsdSB(rB } \mathrm{mB}^{-}\right) \lambda(\mathrm{DE} 3 \text { [lacI lacUV5-T7p07 ind1 } \\
\text { sam7 nin5]) }\end{array}$ & Invitrogen \\
\hline Lactobacillus plantarum WCFS1 & Bifunctional GSH1 synthetase gene $(g s h F) ; 2,256$ bp & Our laboratory \\
\hline Lactobacillus casei WCFS1 & Bifunctional GSH synthetase gene $(g s h F) ; 1,965$ bp & Xiong et al. (2017) \\
\hline pET24a & Protein expression vector of E. coli, kanamycin resistance & Invitrogen \\
\hline
\end{tabular}

${ }^{1} \mathrm{GSH}=$ reduced glutathione. 
LC2W were cultured in de Man, Rogosa and Sharpe medium under anaerobic conditions at $37^{\circ} \mathrm{C}$ (Xiong et al., 2017).

\section{Mining and Phylogenetic Analyses of GshF Based on the Genomes of Lactobacillus}

The complete genome sequences of 20 representative Lactobacillus species, including L. acidophilus NCFM (NC_006814), L. amylovorus GRL 1112 (NC_014724), L. brevis ATCC 367 (NC_008497), L. buchneri NRRL B-30929 (NC_015428), L. casei LC2W (NC_017473), L. crispatus ST1 (NC_014106), L. delbrueckii bulgaricus ATCC 11842 (NC_008054), L. fermentum CECT 5716 (NC_017465), L. gasseri ATCC 33323 (NC_008530), L. helveticus CNRZ32 (NC_021744), L. johnsonii DPC 6026 (NC_017477), L. kefiranofaciens ZW3 (NC_015602), L. paracasei 8700:2 (NC_022112), L. plantarum WCFS1 (NC_004567), L. reuteri DSM 20016 (NC_009513), L. rhamnosus ATCC 8530 (NC_017491), L. ruminis ATCC 27782 (NC_015975), L. sakei 23K (NC_007576), L. salivarius CECT 5713 (NC_017481), and L. sanfranciscensis TMW 1.1304 (NC_015978), were downloaded from NCBI (https:// www.ncbi.nlm.nih.gov) and used to construct a local database using Bioedit software (http://www.mbio .ncsu.edu/bioedit/bioedit.html). Mining of GshF was performed by using Blast (BLOSUM62 scoring matrix) searches of a local database of Lactobacillus to identify sequences homologous to the reported functional GshF from L. plantarum and S. thermophilus (Li et al., 2011;
Ge et al., 2012) with a minimum $25 \%$ identity and an e-value of $1^{-15}$ or lower. The respective sequences were extracted from the local database using Bioedit software. Amino acid sequences of GshF were aligned and used to construct a phylogenetic tree using a neighborjoining algorithm with bootstrap replication of 1,000 in Mega6 software (Tamura et al., 2013).

\section{Plasmid Construction}

All molecular manipulations including restriction enzyme digestions, gene cloning, plasmid transformation, and protein expression were performed as described by Sambrook and Russell (2001). The gshF genes from L. plantarum and L. casei (designated as gshF-Lp and $g s h F-L c$, respectively) were cloned by PCR amplification using primers $g s h F-L p-\mathrm{F} / g s h F-L p-\mathrm{R}$ and $g s h F-L c-$ $\mathrm{F} / g s h F-L c-\mathrm{R}$, respectively. The purified PCR products of $g s h F$ were excised by $N d e \mathrm{I}$ and $X h o \mathrm{I}$ and inserted into plasmid pET24a to create pZX12 and pZX08, respectively.

\section{Fermentation of Engineered E. coli for GshF Expression and GSH Production}

Plasmids pZX12, pZX08, and pET24a (the control) were transformed into $E$. coli BL21(DE3). The clones were grown in Luria-Bertani medium at $37^{\circ} \mathrm{C}$ overnight and preserved at $-80^{\circ} \mathrm{C}$ using $30 \%$ glycerol in $1: 1$ ratio with the cultures as frozen stock cultures. The frozen seeds were inoculated by a 1:100 dilution in a $250-\mathrm{mL}$

Table 2. Primers used in this study

\begin{tabular}{|c|c|c|}
\hline Primer $^{1}$ & Sequence $^{2}\left(5^{\prime} \rightarrow 3^{\prime}\right)$ & Restriction enzyme \\
\hline$g s h F-L p-\mathrm{R}$ & CCGCTGA GATCTTCATTTTTAAACAATGCATCCAACAA & XhoI \\
\hline$g s h F-L c-F$ & GGAATTCCATATGATGTTGAATCATTATTGGCAACT & NdeI \\
\hline$G G C S$-realtime-F & CTGACTTTCTCTGCCCGTATC & \\
\hline$G G C S$-realtime-R & TCGGCTACAAAATCCTCTTCG & \\
\hline$G S$-realtime-F & CCCATCGCAAACATCAACATC & \\
\hline$G P x$-realtime- $\mathrm{R}$ & TCTCCAACTGCTCATATTGCG & \\
\hline$G R$-realtime-F & ACTATGATTACATCGCCATCGG & \\
\hline$G R$-realtime-R & CACAGCCAACATTTACGCAG & \\
\hline gltB-realtime-F & TTTATCTGGATCTTGCGGACC & \\
\hline gltB-realtime- $\mathrm{R}$ & GTACGGTGTTAGTGGAGAAGC & \\
\hline cysK-realtime-F & ACCATGAGTATTGAACGCCG & \\
\hline cysK-realtime-R & AGGTATTTCTCTGGATTGCTGG & \\
\hline$k b l$-realtime-R & TGGCATAGCGATAGCGTTTAG & \\
\hline
\end{tabular}

${ }^{1} \mathrm{~F}=$ forward; $\mathrm{R}=$ reverse.

${ }^{2}$ Underlining denotes site of restriction enzyme. 
Erlenmeyer flask containing $30 \mathrm{~mL}$ of production medium. Isopropyl $\beta$-D-1-thiogalactopyranoside (IPTG) was initially added to $0.1 \mathrm{mM}$ and the cultures were incubated at $37^{\circ} \mathrm{C}$ and $250 \mathrm{rpm}$ for $6 \mathrm{~h}$. The expression and molecular weights of GshF in E. coli were estimated by SDS-PAGE. The IPTG and addition time of IPTG as well as the precursors Gly, L-Glu, and L-Cys were tested to determine the optimal concentrations of each component for GSH production.

\section{RNA Preparation and RT-qPCR Analysis}

The E. coli cells from fermentation medium were collected and total RNA was isolated with an RNA isolation kit (Axygen Biosciences). Genome DNA was removed by digestion with DNase I (Takara) and verified by PCR analysis with the RNA as the template. The quantification and integrity of RNA were determined by both Nanodrop spectrophotometer (Thermo Fisher Scientific, Waltham, MA) and agarose gel electrophoresis. Data were verified in 3 independent experiments. The cDNA was prepared by PrimeScriptRT reagent kit (Takara) and used as template for RT-qPCR, which was performed with a new Lightcycle 96 real-time PCR system (Roche Diagnostics, Risch-Rotkreuz, Switzerland) using an SYBR Premix Ex TaqII kit (Takara). The primers of RT-qPCR are listed in Table 2. The data of each gene were analyzed and normalized using $2^{-\Delta \Delta \mathrm{CT}}$ method (Livak and Schmittgen, 2001) with the $\operatorname{clp} B$ gene encoding $\mathrm{ClpB}$ chaperone as an internal control (Wang et al., 2015).

\section{Analytical Methods}

Cell growth was measured by optical density (OD) at $600 \mathrm{~nm}$ with a UV-visible spectrophotometer (UV1600PC, Mapada Ltd., Shanghai, China). The cells of fermentation broths were harvested by centrifugation at $13,201 \times g$ for $1 \mathrm{~min}$. The cell pellets were resuspended and sonicated for $20 \mathrm{~min}$. The cell lysate was centrifuged at 12,000 $\mathrm{rpm}$ for $10 \mathrm{~min}$ and analyzed by SDS-PAGE. The gel was stained with Coomassie Brilliant Blue staining (R250) and was used to determine protein expression by a low-molecular-weight protein marker (Takara) using an image analysis system (BioRad, Hercules, CA).

Ten milliliters of the culture broth was centrifuged at 12,000 rpm for $1 \mathrm{~min}$, and the supernatant was decanted for GSH assay. The GSH was extracted from E. coli cells by $40 \%$ ethanol and detected with the alloxan method (Xiong et al., 2015). Reduced glutathione reacted with alloxan $(1 \mathrm{~g} / \mathrm{L})$ in a solution of glycine $\left(\begin{array}{ll}0.1 & M\end{array}\right)$ and $\mathrm{NaHPO}_{4}-\mathrm{NaH}_{2} \mathrm{PO}_{4}$ buffer solution $(0.24$ $M, \mathrm{pH}$ 7.6) for $20 \mathrm{~min}$ and was determined by $\mathrm{OD}_{305}$ with UV spectrophotometry. Specific GSH yield (\%) was calculated by GSH concentration/ $\mathrm{OD}_{600}$. All of the assays and experiments were performed in triplicate for each $E$. coli strain with the same transformant.

\section{RESULTS AND DISCUSSION}

\section{Distribution of GshF in Lactobacillus Genomes}

The distribution of putative gshF was examined by genomic analysis of the complete genomes of 20 representative Lactobacillus species. In silico analysis revealed the presence of putative gshF in 11 Lactobacillus species, including L. brevis, L. buchneri, L. casei, L. fermentum, L. paracasei, L. plantarum, L. reuteri, L. rhamnosus, L. ruminis, L. sakei, and L. salivarius (Figure 2A). The domain analysis of 19 putative GshF using the NCBI conserved domain search tool confirmed that all putative GshF possessed the domain of the PRK02471 superfamily (accession no. cl25875) responsible for bifunctional $\gamma$-GCS/GS, suggesting that they have the potential to synthesize GSH using the precursor AA. The presence of putative $g s h F$ in genomes indicated the ability of GSH biosynthesis in Lactobacillus, which directly demonstrated why many Lactobacillus species could produce GSH (Pophaly et al., 2012). In addition, L. buchneri, L. fermentum, $L$. plantarum, and $L$. reuteri have multiple gene numbers of $g s h F$ (designated $g s h F-n, \mathrm{n} \geq 2$ ) distributed in the genomes, suggesting that they may be highly resistant to extreme environments due to the stronger ability of GSH biosynthesis.

Phylogenetic tree analysis showed that GshF in Lactobacillus species could be divided into 3 distinct types that had low sequence identities and longer genetic distances but were highly conserved in evolution (Figure 2B). For evolution distances, GshF in Lactobacillus were located relatively close to $\gamma$-GCS of E. coli, not GS of E. coli, implying that evolutionary GS may appear earlier than $\gamma$-GCS and GshF (Copley and Dhillon, 2002). The GshF-n in the same Lactobacillus species showed low identities with each other and had longer distances to each other. For example, 4 GshF (from GshF-1 to GshF-4) were discovered from the genome of L. fermentum; their sequence identities were less than $50 \%$ with each other using NCBI Blastp search (https://blast.ncbi.nlm.nih.gov/Blast.cgi).

\section{Heterologous Expression of GshF from Lactobacillus in E. coli}

The majority of GshF in Lactobacillus (12/19 GshF) were assigned to type I based on a phylogenetic tree (Figure 2B). Thus, type I GshF from L. plantarum and 
L. casei (GshF-Lp and GshF-Lc) were selected as the model GshF for functional analysis. The genes $g s h F-L p$ and $g s h F-L c$ were cloned from $L$. plantarum WCFS1 and $L$. casei LC2W by PCR and inserted into the expression vector pET24a to create pZX12 and pZX08 (Figure 3). The plasmids were sequenced by Sangon Biotech (Shanghai, China), showing that gshF-Lp and $g s h F-L c$ with high identities of $100 \%$ using NCBI Blastx analysis were successfully inserted into pET24a. When pZX12 and pZX08 were transformed into the ex- pression host E. coli BL21(DE3), SDS-PAGE was used to analyze the protein expression. Using Vector NTI Advance software (Invitrogen, Carlsbad, CA), GshFLp and GshF-Lc had theoretical molecular weights of 83.1 and $71.3 \mathrm{kDa}$ as well as isoelectric points of 6.65 and 5.85, respectively. Compared with the control $E$. coli harboring empty vector $\mathrm{pET} 24 \mathrm{a}, E$. coli containing pZX12 or pZX08 had a desired band at 70 to $85 \mathrm{kDa}$, suggesting that $E$. coli could significantly express heterologous GshF proteins from Lactobacillus (Figure 4A).

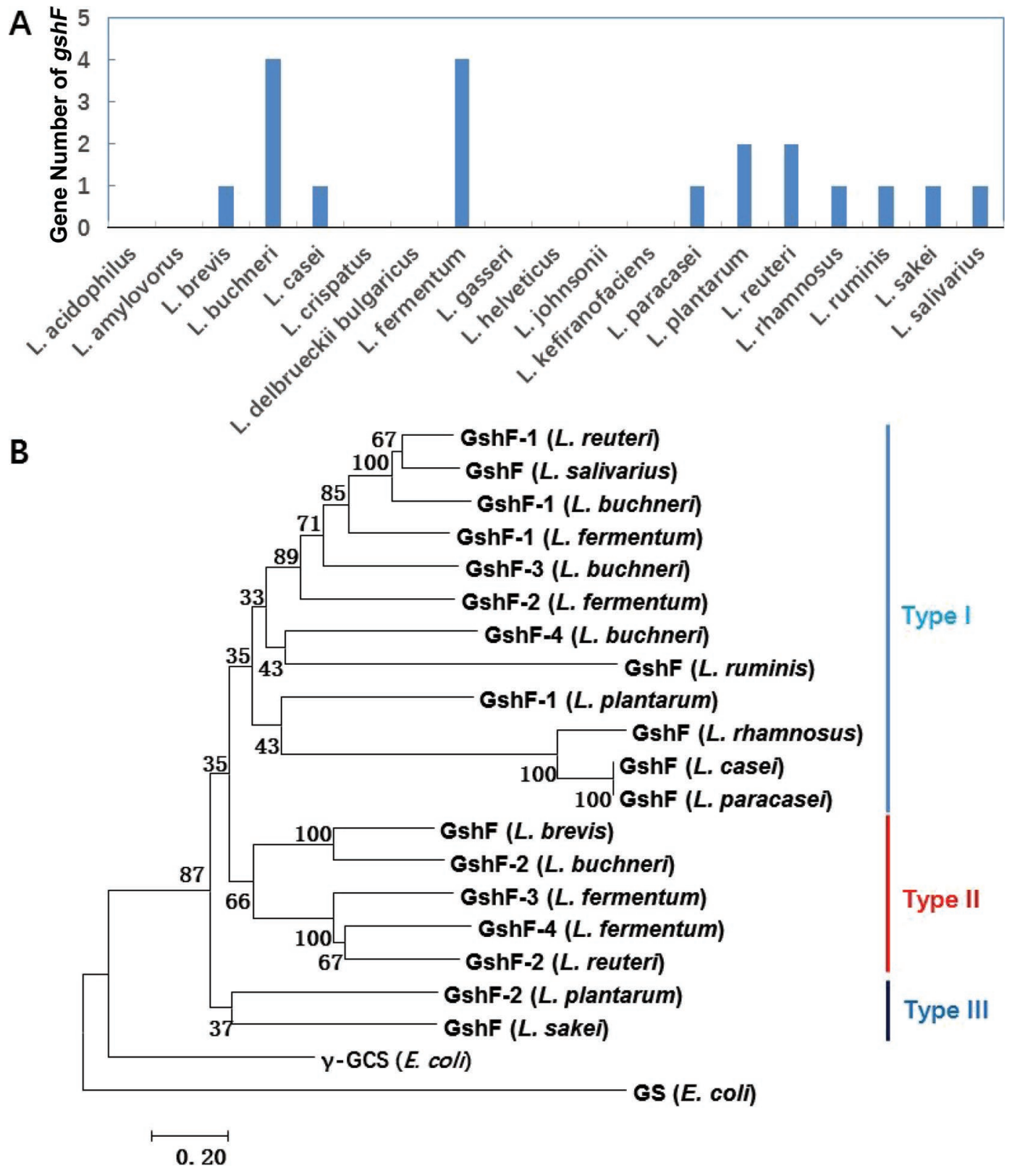

Figure 2. Presence and phylogenetic analysis of bifunctional glutathione synthetase (GshF) in representative Lactobacillus genomes. (A) Gene number of gshF in 20 selected Lactobacillus species. (B) Phylogenetic analysis of GshF in Lactobacillus. The protein sequences were used to construct a phylogenetic tree using a neighbor-joining algorithm with bootstrap replication of 1,000 with $\gamma$-glutamylcysteine synthetase ( $\gamma$-GCS) and reduced glutathione synthase (GS) of Escherichia coli BL21(DE3) as the control. Color version available online. 


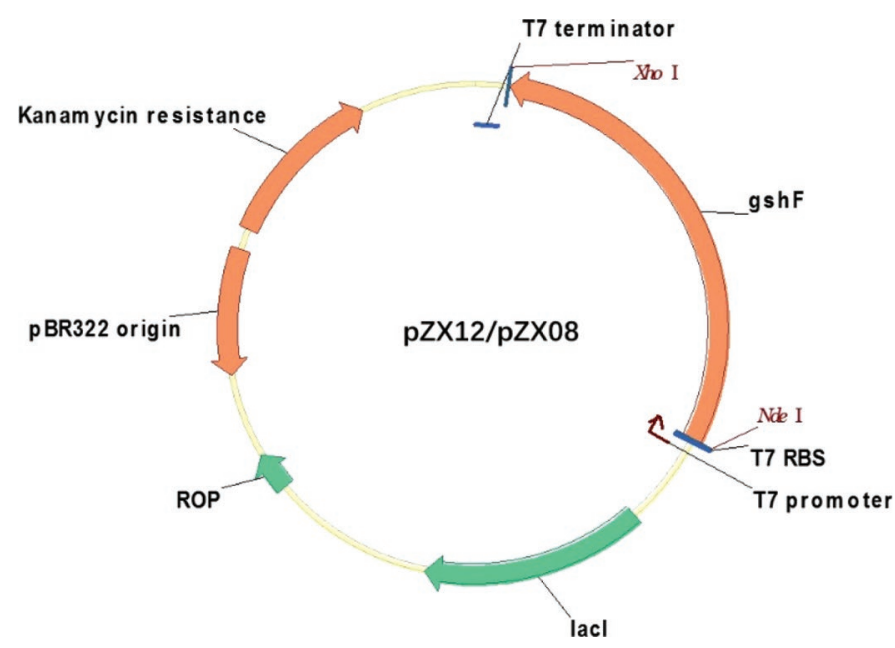

Figure 3. Schematic representation of the recombinant expression vectors containing the $g s h F$ genes from Lactobacillus plantarum WCFS1 and Lactobacillus casei LC2W. Color version available online.

The feedback inhibition of $\gamma$-GCS activity by GSH at both the transcriptional and posttranslational levels is considered to be a biochemcial mechanism that prevents the overaccumulation of GSH in living cells ( Ge et al., 2012). To overcome this problem in GSH production, a strategy with the use of GshF that is insensitive to GSH feedback inhibition was developed to improve the rate of GSH biosynthesis even in the presence of a high concentration of GSH (Wang et al., 2011, 2016; Ge et al., 2012; Yang et al., 2016). Hence, to demonstrate that GshF-Lp and GshF-Lc were functionally expressed in E. coli, GSH biosynthesis was examined in this work.
If GSH concentration was significantly improved in cells with expressing GshF, it indicated functional expression and strong activities of GshF. For intracellular GSH titer, E. coli harboring pZX12 and pZX08 had 103.1 and $96.7 \mu M$ GSH, respectively, in comparison with $E$. coli/pET24a (8.3 $\mu M$ GSH, the control). Hence, GshF definitely enhanced GSH biosynthesis in E. coli, suggesting that GshF from Lactobacillus had functional activities for GSH biosynthesis (Figure 4B).

\section{Effects of Culture Condition on GSH Biosynthesis in E. coli Expressing GshF from Lactobacillus}

Vast literature has reported that culture condition (i.e., induction condition) could strongly affect protein expression and activity and eventually affect the titer of target product (Fucinos et al., 2014). In this study, E. coli/pZX12 containing $g s h F-L p$ had higher GSH titer than E. coli/pZX08 containing gshF-Lc. Therefore, E. coli/pZX12 was used for the next experiments to examine whether GSH biosynthesis could further improve by affecting GshF expression with the change of culture condition. For the expression of recombinant proteins using the pET-serial vectors with $\mathrm{T} 7$ promoter, $1 \mathrm{mM}$ IPTG is recommended for full induction; however, proper induction was generally obtained at low IPTG concentrations (e.g., $0.01 \mathrm{~m} M$; Fucinos et al., 2014). Thus, the effects of induction conditions such as IPTG concentration and induction time were investigated in this study. The cells were initially induced with a range of IPTG concentrations between 0.1 and $1 \mathrm{~m} M$. There was a marked increase in the GSH titer when IPTG

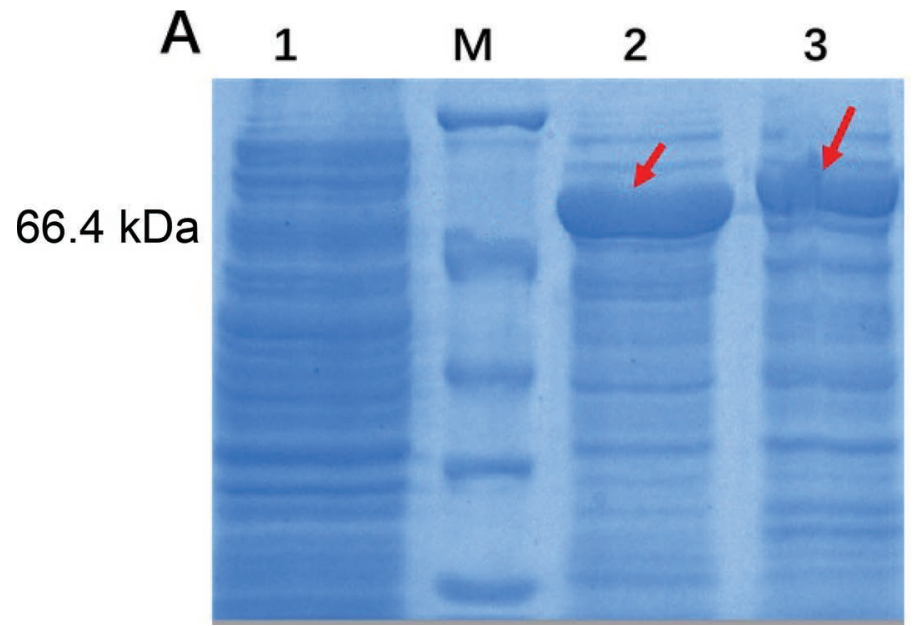

B

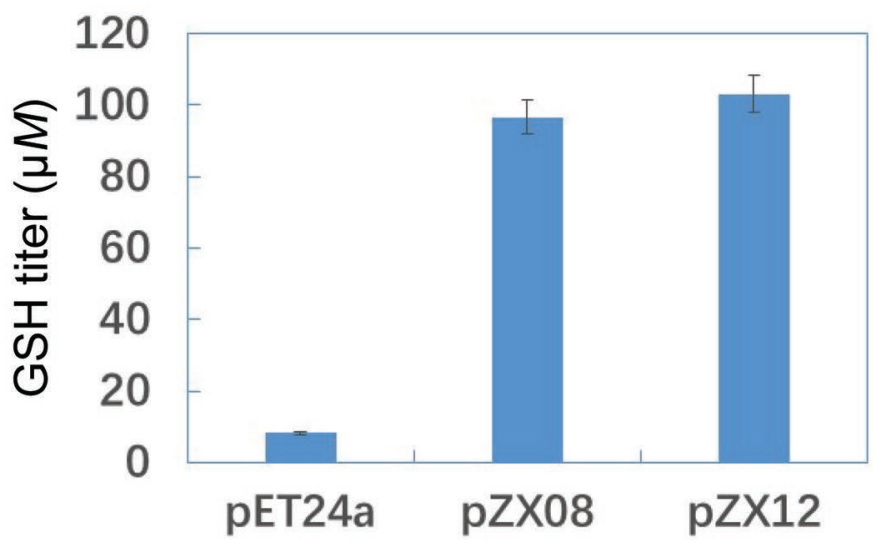

Figure 4. Expression of bifunctional glutathione synthetase (GshF) and reduced glutathione (GSH) titer in Escherichia coli BL21(DE3). (A) Expression of GshF by SDS-PAGE. Lane $\mathrm{M}=$ low-molecular-weight marker (Takara Biomedical Technology Co. Ltd., Beijing, China); lane 1 = control E. coli BL21(DE3)/pET24a; lane $2=$ E. coli BL21(DE3)/pZX08; lane $3=E$. coli BL21(DE3)/pZX12. Arrows indicate the expression of GshF. (B) The GSH titers in E. coli BL21(DE3)/pZX12 and E. coli BL21(DE3)/pZX08 when isopropyl $\beta$-D-1-thiogalactopyranoside was initially added to $0.1 \mathrm{mM}$ and the cultures were incubated at $37^{\circ} \mathrm{C}$ and $250 \mathrm{rpm}$ for $6 \mathrm{~h}$. Error bars indicate SD of the mean of triplicate. Color version available online. 

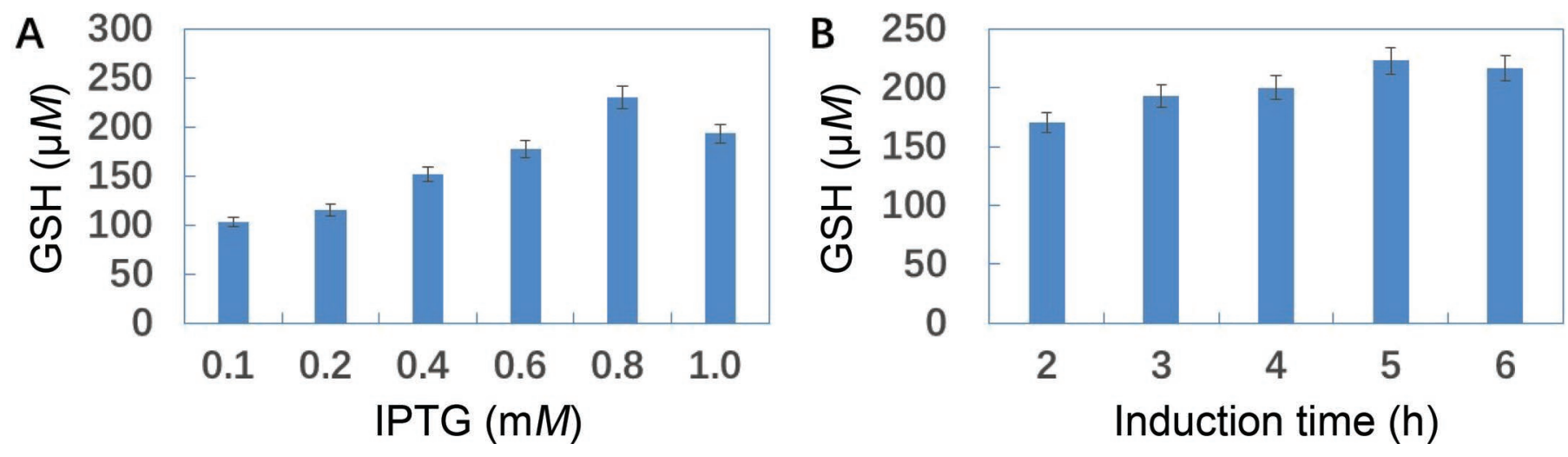

Figure 5. The effects of (A) isopropyl $\beta$-D-1-thiogalactopyranoside (IPTG) concentration and (B) induction time on reduced glutathione (GSH) production in Escherichia coli BL21(DE3)/pZX12. Error bars indicate SD of the mean of triplicate. Color version available online.

concentration was increased from 0.1 to $0.8 \mathrm{mM}$ (Figure $5 \mathrm{~A})$. The highest yield of GSH $(220 \mathrm{mM})$ was reached with $0.8 \mathrm{~m} M$ IPTG. Various induction time between 2 and $6 \mathrm{~h}$ was also investigated for GSH production. It was apparent that $5 \mathrm{~h}$ of induction time gave the highest GSH titer (Figure 5B).

The addition of GSH precursors Gly, L-Glu, and L-Cys can significantly stimulate GSH biosynthesis (Xiong et al., 2015). Yield of GSH could also gradually improve with the increasing concentration of precursors (Fei et al., 2009; Wang et al., 2012). For example, using Pichia pastoris harboring $\gamma$-GCS and GS of GSH biosynthetic pathway from $S$. cerevisiae, GSH titer was obviously improved from $2.03 \mathrm{~g} / \mathrm{L}$ to $4.15 \mathrm{~g} / \mathrm{L}$ when the precursor concentration was increased from 5 to 15 $\mathrm{mmol} / \mathrm{L}$ (Fei et al., 2009). Various concentrations of Gly, L-Glu, and L-Cys ranging from 0 to $20 \mathrm{mM}$ were initially added into the medium for GSH biosynthesis in this work (Figure 6). When precursor concentration was increased from 0 to $5 \mathrm{~m} M$, cell density was almost not changed, but GSH titer and specific GSH yield were significantly increased. Cell density and GSH titer were gradually decreased with the increase of precursor concentration from 5 to $20 \mathrm{mM}$; cell growth was inhibited at $20 \mathrm{~m} M$ precursor (Figure 6A). The highest GSH titer and specific GSH yield were 286.5 $\mu M$ and 173.3 $\mu M / \mathrm{OD}_{600}$ (Figure $6 \mathrm{~B}$ and $6 \mathrm{C}$ ), which were improved by 27.9 and $31.9 \%$, respectively, in comparison with the control (without addition of precursors).

\section{Transcriptional Analysis of GSH Metabolism and Precursor Biosynthesis in E. coli Expressing GshF from Lactobacillus}

To our knowledge, there is no report on the systemic effect of heterologous expression of GshF in the host. Hence, to assess how GshF expression affects endogenous metabolism in E. coli, transcriptional change
A

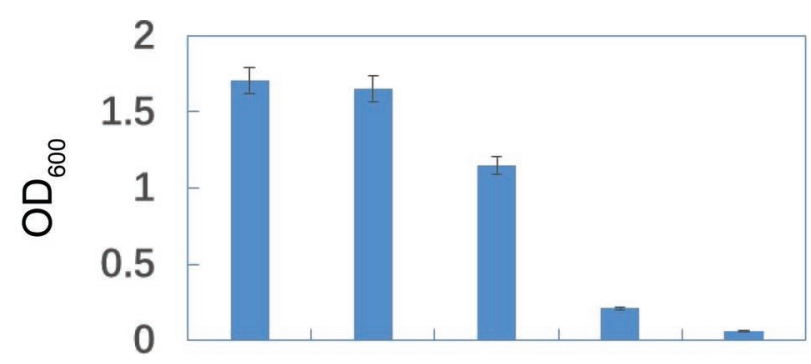

B
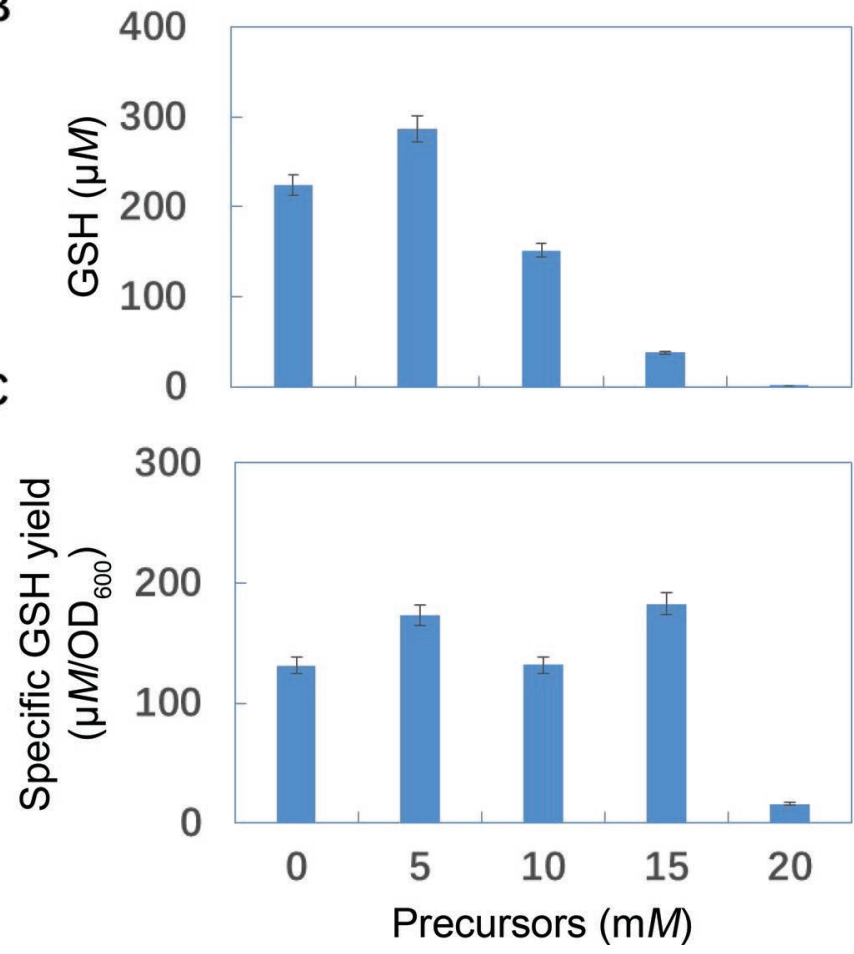

Figure 6. The effect of reduced glutathione (GSH) precursors on (A) cell growth, (B) GSH titer, and (C) specific GSH yield in Escherichia coli BL21(DE3)/pZX12. OD = optical density. Error bars indicate SD of the mean of triplicate. Color version available online. 

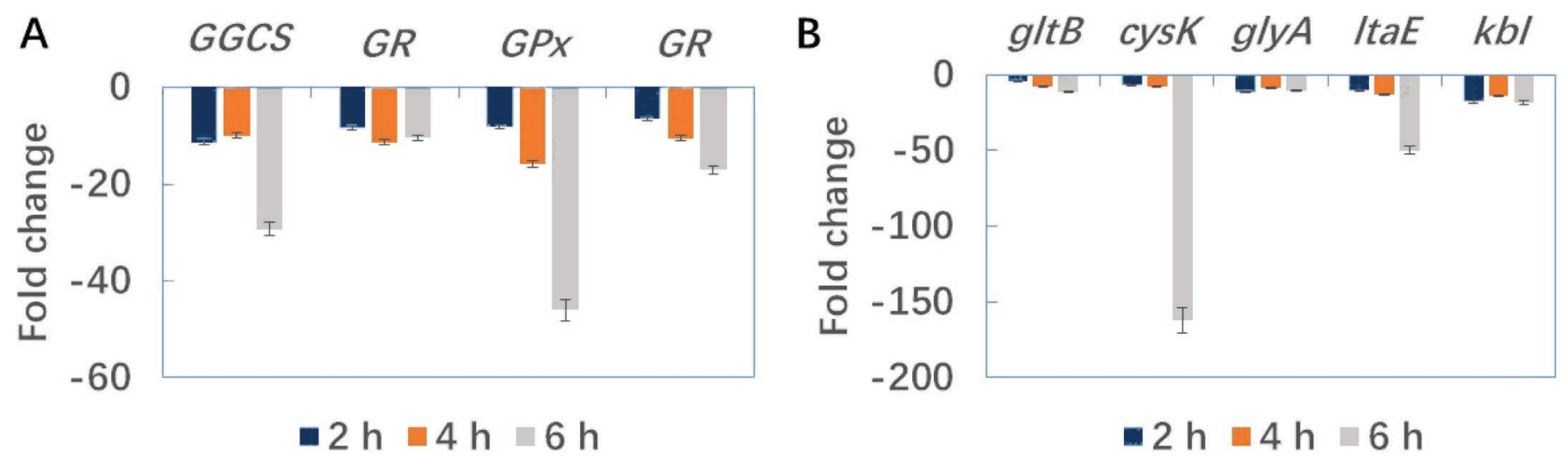

Figure 7. The transcriptional change in the key genes of (A) reduced glutathione (GSH) metabolism and (B) GSH precursor (Gly, L-Glu, and L-Cys) biosynthesis in Escherichia coli BL21(DE3)/pZX12 in comparison with the control strain BL21(DE3)/pET24a. Error bars indicate $\mathrm{SD}$ of the mean of triplicate. Color version available online.

of the key genes of endogenous GSH metabolism and GSH precursors Gly, L-Glu, and L-Cys biosynthesis was investigated by RT-qPCR. Compared with the control E. coli/pET24a (without expressing GshF), all genes of endogenous GSH metabolism ( $G G C S, G S, G P x$, and $G R$ ) were clearly suppressed more than 5-fold at the transcriptional level in E. coli/pZX12 (Figure $7 \mathrm{~A}$ ), suggesting that GshF expression resulted in the decrease of endogenous GSH biosynthesis. It also may be partly due to the feedback inhibition of high GSH concentration. Moreover, it further showed that high GSH titer in E. coli/pZX12 should be attributed to heterologous expression of GshF. Interestingly, the key genes of GSH precursor biosynthesis - gltB (responsible for glutamate synthase, large subunit), cysK (responsible for cysteine synthase A), glyA (responsible for serine hydroxymethyltransferase), ltaE (responsible for L-allo-threonine aldolase), and $k b l$ (responsible for glycine C-acetyltransferase) - were also strongly inhibited more than 4-fold in E. coli/pZX12 (Figure 7B). Notable was the $>130$-fold suppression of cysK, the key gene for cysteine biosynthesis, at $6 \mathrm{~h}$. Our result indicated that GshF expression reduces or even inhibits the biosynthesis of precursor AA. It seemed contradictory to high GSH titer in E. coli/pZX12, but it was reasonable by the effect of exogenous addition of abundant precursors. To our knowledge, this is the first report about the effect of heterologous expression of GshF on endogenous metabolism in the host.

Based on the literature (Li et al., 2011; Hara et al., 2012; Zhang et al., 2016b), construction of the high-yield strains is important to GSH biosynthesis. Except engineered target genes of GSH and precursor metabolism [i.e., GGCS, GS, GPx, GR, ggt (encoding glutathione hydrolase), рерT (encoding peptidase $\mathrm{T}$ ), $s d a A$ (encoding L-Ser deaminase I), MET14 (encoding adenylylsulfate kinase), MET16 (encoding 3'-phosphoadenylsulfate reductase), and CYS4 (encoding cystathionine $\beta$-synthase) in the host (Suzuki et al., 2011; Ge et al., 2012; Hara et al., 2012; Zhang et al., 2016b)], our study showed that heterologous expression of GshF could be an efficient strategy for constructing microbial cell factories with high GSH yield. In recent years, GSH have been reported to protect LAB against various environmental stresses (Lee et al., 2010; Zhang et al., 2010, 2012, 2016a). Therefore, overexpression of $g s h F$ could provide a useful method for the increased viability and productivity of LAB during industrial processes and gastrointestinal transit.

\section{CONCLUSIONS}

Nineteen putative $g s h F$ genes were mined from representative Lactobacillus genomes in this study, indicating that $g s h F$ is enriched in Lactobacillus. To characterize these putative GshF, GshF-Lp and GshF-Lc were selected and overexpressed in E. coli BL21(DE3). Our result showed the functional activities of putative GshF from Lactobacillus, which could remarkably improve GSH biosynthesis. Compared with the control, GSH titer $(286.5 \mu M)$ was significantly increased by $177.9 \%$ through optimizing induced condition and precursor concentration. Based on RT-qPCR analysis, the key genes of GSH metabolism and precursor biosynthesis were strongly inhibited by heterologous GshF expression, suggesting that the increase of GSH titer was attributed to GshF expression.

\section{ACKNOWLEDGMENTS}

This work was supported by the National Natural Science Foundation of China (grant no. 31771956 and 
31100073), "Shuguang Program" by Shanghai Education Development Foundation and Shanghai Municipal Education Commission (no. 15SG42), and Key Laboratory of Synthetic Biology, Chinese Academy of Sciences, Shanghai, China.

\section{REFERENCES}

Copley, S. D., and J. K. Dhillon. 2002. Lateral gene transfer and parallel evolution in the history of glutathione biosynthesis genes. Genome Biol. 3:1-16.

Fei, L., Y. Wang, and S. Chen. 2009. Improved glutathione production by gene expression in Pichia pastoris. Bioprocess Biosyst. Eng. 32:729-735.

Fucinos, P., E. Atanes, O. Lopez-Lopez, M. Solaroli, M. E. Cerdan, M. I. Gonzalez-Siso, L. Pastrana, and M. L. Rua. 2014. Cloning, expression, purification and characterization of an oligomeric Histagged thermophilic esterase from Thermus thermophilus HB27. Process Biochem. 49:927-935.

Ge, S., T. Zhu, and Y. Li. 2012. Expression of bacterial GshF in Pichia pastoris for glutathione production. Appl. Environ. Microbiol. 78:5435-5439.

Hara, K. Y., K. Kiriyama, A. Inagaki, H. Nakayama, and A. Kondo. 2012. Improvement of glutathione production by metabolic engineering the sulfate assimilation pathway of Saccharomyces cerevisiae. Appl. Microbiol. Biotechnol. 94:1313-1319.

Kleerebezem, M., J. Boekhorst, R. van Kranenburg, D. Molenaar, O. P. Kuipers, R. Leer, R. Tarchini, S. A. Peters, H. M. Sandbrink, M. W. Fiers, W. Stiekema, R. M. Lankhorst, P. A. Bron, S. M. Hoffer, M. N. Groot, R. Kerkhoven, M. de Vries, B. Ursing, W. M. de Vos, and R. J. Siezen. 2003. Complete genome sequence of Lactobacillus plantarum WCFS1. Proc. Natl. Acad. Sci. USA 100:1990-1995.

Lee, K., K. Pi, E. B. Kim, B. S. Rho, S. K. Kang, H. G. Lee, and Y. J. Choi. 2010. Glutathione-mediated response to acid stress in the probiotic bacterium, Lactobacillus salivarius. Biotechnol. Lett. 32:969-972.

Li, W., Z. Li, J. Yang, and Q. Ye. 2011. Production of glutathione using a bifunctional enzyme encoded by gshF from Streptococcus thermophilus expressed in Escherichia coli. J. Biotechnol. 154:261268.

Li, Y., J. Hugenholtz, W. Sybesma, T. Abee, and D. Molenaar. 2005. Using Lactococcus lactis for glutathione overproduction. Appl. Microbiol. Biotechnol. 67:83-90.

Li, Y., G. Y. Wei, and J. Chen. 2004. Glutathione: A review on biotechnological production. Appl. Microbiol. Biotechnol. 66:233-242.

Livak, K. J., and T. D. Schmittgen. 2001. Analysis of relative gene expression data using real-time quantitative PCR and the 2(-Delta Delta C(T)) method. Methods 25:402-408.

Pophaly, S. D., S. Poonam, S. D. Pophaly, S. Kapila, D. K. Nanda, S. K. Tomar, and R. Singh. 2017. Glutathione biosynthesis and activity of dependent enzymes in food grade lactic acid bacteria harboring multidomain bifunctional fusion gene $(g s h F)$. J. Appl. Microbiol. https://doi.org/10.1111/jam.13471.

Pophaly, S. D., R. Singh, S. D. Pophaly, J. K. Kaushik, and S. K. Tomar. 2012. Current status and emerging role of glutathione in food grade lactic acid bacteria. Microb. Cell Fact. 11:114.

Sambrook, J., and D. W. Russell. 2001. Molecular Cloning: A Laboratory Manual. Cold Spring Harbor Laboratory Press, Cold Spring Harbor, NY.

Settachaimongkon, S., H. J. van Valenberg, I. Gazi, M. J. Nout, T. C. van Hooijdonk, M. H. Zwietering, and E. J. Smid. 2016. Influence of Lactobacillus plantarum WCFS1 on post-acidification, metabolite formation and survival of starter bacteria in set-yoghurt. Food Microbiol. 59:14-22.

Siezen, R. J., C. Francke, B. Renckens, J. Boekhorst, M. Wels, M. Kleerebezem, and S. A. van Hijum. 2012. Complete resequencing and reannotation of the Lactobacillus plantarum WCFS1 genome. J. Bacteriol. 194:195-196.

Stout, J., D. De Vos, B. Vergauwen, and S. N. Savvides. 2012. Glutathione biosynthesis in bacteria by bifunctional GshF is driven by a modular structure featuring a novel hybrid ATP-grasp fold. J. Mol. Biol. 416:486-494.

Suzuki, T., A. Yokoyama, T. Tsuji, E. Ikeshima, K. Nakashima, S. Ikushima, C. Kobayashi, and S. Yoshida. 2011. Identification and characterization of genes involved in glutathione production in yeast. J. Biosci. Bioeng. 112:107-113.

Tamura, K., G. Stecher, D. Peterson, A. Filipski, and S. Kumar. 2013. MEGA6: Molecular Evolutionary Genetics Analysis version 6.0. Mol. Biol. Evol. 30:2725-2729.

Vergauwen, B., D. De Vos, and J. J. Van Beeumen. 2006. Characterization of the bifunctional gamma-glutamate-cysteine ligase/ glutathione synthetase (GshF) of Pasteurella multocida. J. Biol. Chem. 281:4380-4394.

Wang, D., C. Wang, H. Wu, Z. Li, and Q. Ye. 2016. Glutathione production by recombinant Escherichia coli expressing bifunctional glutathione synthetase. J. Ind. Microbiol. Biotechnol. 43:45-53.

Wang, G., S. Yin, H. An, S. Chen, and Y. Hao. 2011. Coexpression of bile salt hydrolase gene and catalase gene remarkably improves oxidative stress and bile salt resistance in Lactobacillus casei. J. Ind. Microbiol. Biotechnol. 38:985-990.

Wang, J., Z. Xiong, S. Li, and Y. Wang. 2015. Exploiting exogenous MEP pathway genes to improve the downstream isoprenoid pathway effects and enhance isoprenoid production in Escherichia coli. Process Biochem. 50:24-32.

Wang, Y., D. Wang, G. Wei, and N. Shao. 2012. Enhanced co-production of S-adenosylmethionine and glutathione by an ATP-oriented amino acid addition strategy. Bioresour. Technol. 107:19-24.

Xiong, Z.-Q., M.-J. Guo, J. Chu, Y.-P. Zhuang, and S.-L. Zhang. 2015. On-line specific growth rate control for improving reduced glutathione production in Saccharomyces cerevisiae. Biotechnol. Bioprocess Eng. 20:887-893.

Xiong, Z. Q., Q. H. Wang, L. H. Kong, X. Song, G. Q. Wang, Y. J. Xia, H. Zhang, Y. Sun, and L. Z. Ai. 2017. Improving the activity of bile salt hydrolases in Lactobacillus casei based on in silico molecular docking and heterologous expression. J. Dairy Sci. 100:975-980.

Yang, J., W. Li, D. Wang, H. Wu, Z. Li, and Q. Ye. 2016. Characterization of bifunctional L-glutathione synthetases from Actinobacillus pleuropneumoniae and Actinobacillus succinogenes for efficient glutathione biosynthesis. Appl. Microbiol. Biotechnol. 100:6279-6289.

Zhang, J., G. C. Du, Y. Zhang, X. Y. Liao, M. Wang, Y. Li, and J. Chen. 2010. Glutathione protects Lactobacillus sanfranciscensis against freeze-thawing, freeze-drying, and cold treatment. Appl. Environ. Microbiol. 76:2989-2996.

Zhang, J., Y. Li, W. Chen, G. C. Du, and J. Chen. 2012. Glutathione improves the cold resistance of Lactobacillus sanfranciscensis by physiological regulation. Food Microbiol. 31:285-292.

Zhang, J., Q. Liu, W. Chen, G. Du, and J. Chen. 2016a. Protection of lyophilized milk starter Lactobacillus casei Zhang by glutathione. J. Dairy Sci. 99:1846-1852.

Zhang, J., C. Quan, C. Wang, H. Wu, Z. Li, and Q. Ye. 2016b. Systematic manipulation of glutathione metabolism in Escherichia coli for improved glutathione production. Microb. Cell Fact. 15:38. 